\title{
ANÁLISE DAS OSCILAÇÕES DA TEMPERATURA DO SOLO ATRAVÉS DA TRANSFORMADA DE ONDELETAS
}

\author{
Rayonil G. Carneiro, ,*, Marcos A. L. Moura², Vicente P. R. Silva ${ }^{1}$, Aurilene B. Santos ${ }^{3}$, \\ Antônio M. D. Andrade ${ }^{1}$ \\ 1Universidade Federal de Campina Grande \\ Universidade Federal de Alagoas \\ ${ }^{3}$ Universidade Federal do Rio Grande do Norte \\ *rayonilcarneiro@gmail.com
}

\begin{abstract}
Variations in the soil temperature (TS) inside a fragment of Atlantic forest in the city of Coruripe-AL, were studied using data from a micrometeorological tower of $26 \mathrm{~m}$, installed inside the forest. The data covering the year 2012 were subjected to wavelet transform. This method involves transformation of a number in a unidimensional space of time and frequency, allowing determining the dominant scales of variability and its temporal variations. The results showed that the variability of the TS region is defined by multi-scales located at certain intervals of days. However, the variability is associated with solar radiation $(\mathrm{Rg})$ and precipitation.
\end{abstract}

\section{INTRODUÇÃO}

As flutuações diárias e anuais da temperatura do solo influenciam nos processos biológicos e químicos, nas taxas de decomposição e de mineração da matéria orgânica do solo e a liberação de $\mathrm{CO}_{2}$. É também um dos principais fatores de influência da respiração do solo, particularmente nas florestas tropicais (Graham et. al., 2010).

Atualmente, estudos para poder compreender os fenômenos físicos que estão agindo em qualquer sistema natural exigem abordagens matemáticas robustas. Uma destas ferramentas é a Transformada de Ondeletas, capaz de analisar séries-temporais obtidas de qualquer sistema físico (Bolzan, 2004). Este método envolve transformação de uma série unidimensional em um espaço de tempo e frequência, o que permite determinar as escalas de variabilidade dominantes e suas variações temporais (Silva et. al., 2009). 
Pretende-se nesse trabalho aplicar a transformada de ondeletas nos dados de temperatura do solo no interior de uma floresta de mata atlântica, no intuito de investigar seu comportamento e escalas de oscilações.

\section{MATERIAL E MÉTODOS}

Na realização do presente estudo utilizou-se dados obtidos através de uma torre micrometeorológica de 26 metros de altura (1000'37' 'S, 36 $\left.6^{\circ} 17^{\prime} 60^{\prime \prime} \mathrm{W}\right)$. Esta se localiza na Reserva Particular do Patrimônio Natural (RPPN) pertencente à usina Coruripe Açúcar e Álcool S/A, no município de Coruripe, Alagoas. As medições das temperaturas do solo (TS) foram realizadas com termopares, tipo cobre/constatam, nas profundidades 1,20 e $50 \mathrm{~cm}$, para todo o ano de 2012.

A fim de se identificar possíveis tendências, períodos cíclicos, variação sazonal e aleatória será utilizada a transformada em ondeletas (maiores detalhes em Torrence e Compo (1998)) a partir dos dados observacionais de TS. Essa é uma metodologia de decomposição de sinais que permite detectar tanto a oscilação quanto a localização temporal das componentes do sinal, o que facilita a associação dos períodos observados com os fenômenos atmosféricos.

\section{RESULTADOS E DISCUSSÃO}

Os cálculos da transformada de ondeletas (TO) para os dados de temperatura do solo (TS) no interior da mata atlântica, demonstraram um aquecimento deste nos mesmos períodos em todas as profundidades (Figura 1a i, ii e iii). Observou-se claramente um sinal persistente e bastante homogêneo na escala diária de 24 horas, com GWP (Figura 1b i, ii e iii) apresentando um forte pico de energia associado a essa componente, em função da resposta do solo a radiação solar global $(\mathrm{Rg})$. Notou-se que a partir do dia 150 , inicio do período chuvoso, ocorre uma diminuição da intensidade deste sinal, devido a maior cobertura de nuvens e por consequência a menor incidência de Rg. 
A TS também exibiu núcleos de maior intensidade em torno de 16 - 32 dias com alto grau de ocorrência e homogeneidade, entre os dias 50 - 55 (Fevereiro de 2012), entre 150 - 170 (maio à junho de 2012) e entre 300 - 315 (outubro à novembro de 2012). A GWP mostra energias elevadas para estas oscilações. Esta oscilação pode estar relacionada com a quantidade de água existente no solo para a evaporação, devido ao ano de 2012 ter registrado chuvas abaixo da normal climatológica, com precipitações de $33 \mathrm{~mm}$ em janeiro, 126,6 mm em entre abril e maio e $212,3 \mathrm{~mm}$ entre agosto e setembro, gerando tais periodicidades da TS.

Há também um núcleo pequeno de 8 dias com menor intensidade que os citados acima, o qual está associado com os meses mais secos da região. Os referidos núcleos apresentam maiores intensidades na camada de $1 \mathrm{~cm}$ tendo uma leve redução nas camadas subsequentes $(20$ e $50 \mathrm{~cm})$.

\section{CONCLUSÕES}

A partir da transformada de ondeletas obtiveram-se resultados os quais indicaram que a variabilidade da TS no interior da Mata atlântica para o ano de 2012 foi definida por multi-escalas temporais localizadas em certos intervalos de tempo. Principalmente a variabilidade diária e os ciclos de 16 - 32 dias que apresentaram maiores intensidades. Estes ciclos da TS podem sofrer variações em função da precipitação e do Rg.

\section{REFERÊNCIA BIBLIOGRÁFICA}

Bolzan, M. J. A. Análise da transformada em ondeletas aplicada em sinal geofísico. Revista Brasileira de Ensino de Física, v. 26, n. 1, p. 37-41, 2004.

Graham, E. A.; Lam, Y.; Yuen, E. M. Forest understory soil temperatures and heat flux calculated using a Fourier model and scaled using a digital camera. Agricultural and Forest Meteorology. v. 150, p. 640-649, 2010. 


\section{ciênciaenatura}

Silva, D. F.; Sousa, F. A. S.; Kayano, M. T. Uso de iac e ondeletas para análise da influência das multiescalas temporais na precipitação da bacia do rio mundaú. Revista Engenharia Ambiental, Espírito Santo do Pinhal - SP, v. 6, n. 1, p. 180-195, 2009.

Torrence, C.; Compo, G.P.: A practical guide to wavelet analysis. Bulletin of the American Meteorological Society., v. 79, p. 61-78, 1998.
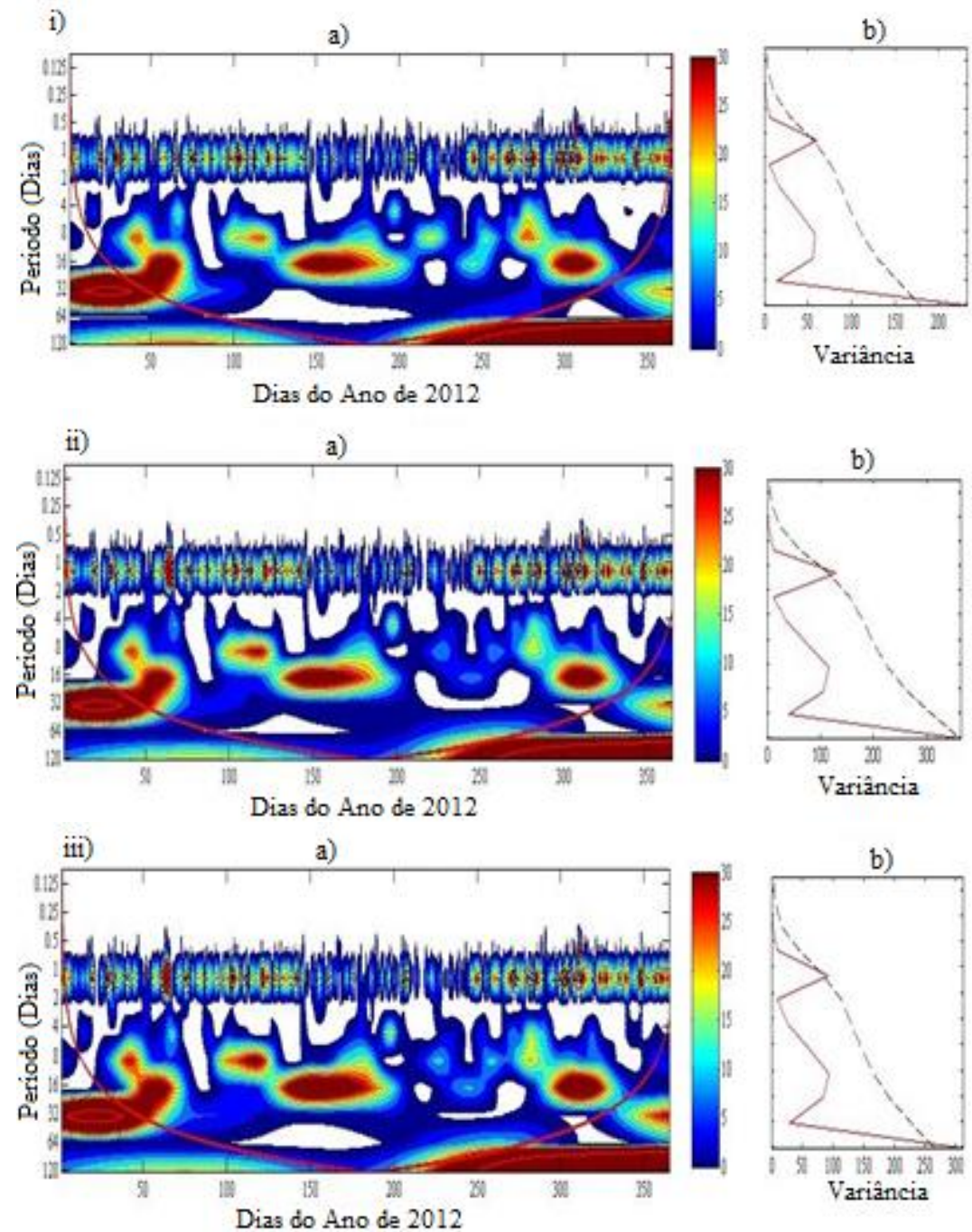

Figura 1 - Espectro de energia da ondeleta (EPW) para a Temperatura do solo nas profundidades de 1 cm (i), $20 \mathrm{~cm}$ (ii) e $50 \mathrm{~cm}$ (iii) (a) e Espectro global de ondeleta (GWP), cujo contorno tracejado indica que o espectro de ondeleta global é significativo ao nível de confiança de $95 \%$, para a mesma variável e profundidades (b). 